\title{
El Sistema Nacional e-México
}

\section{Entre incertidumbres y renovadas esperanzas}

\section{OCTAVIO ISLAS*}

\section{La contribución de Al Gore en el desarrollo}

de los programas de e-Gobierno

1 Gore, vicepresidente de los Estados Uni-
dos en el gobierno de William Clinton,
definitivamente admite ser reconocido como un gran visionario de las posibilidades de internet y como el principal teórico de la ciberpolítica. Gore comprendía la necesidad de emplear las más avanzadas tecnologías de información y comunicaciones (Tic), en la "reinvención del gobierno". Gore encabezó la "Sociedad Nacional para la Reinvención del Gobierno", iniciativa que dio comienzo en 1993 y cuyo propósito principal era garantizar que la aplicación de las avanzadas tecnologías de información y comunicaciones, pudiera incidir positivamente en la transformación de la administración pública.

Gore comprendía que el inteligente empleo de las $\mathrm{\pi c}$, en la administración pública, no sólo permitiría simplificar un considerable número de trámites, el acertado empleo de las TIC, además, podría incidir en la positiva transformación del Estado, volviéndolo más compacto, productivo, abierto y sensible a las necesidades del ciudadano. En el documento "Principios fundamentales de la construcción de una sociedad de la información", Gore identificó los cinco principios rectores de la sociedad de la información. Estos son: inversión privada, competencia, regulación flexible, acceso abierto y servicio universal:

"La Infraestructura Mundial de Información (IMI) es una red masiva de redes de comunicación que cambiará para siempre el modo en el que los ciudadanos de todo el mundo viven, aprenden, trabajan y se comunican entre sí.

- Especialista en comunicación estratégica y cibercultura. Instituto Tecnołógico y de Estudios Superiores de Monterrey, Campus Estado de México. Comeo electrónico: octavio.istasoitesm.mx 
Esta red mundial le permitirá a la aldea más lejana examinar la biblioteca más adelantada. Les permitirá a los médicos de un continente examinar pacientes que vivan en otro. Le ayudará a una familia del Hemisferio Norte a mantenerse en contacto con parientes en el Hemisferio Sur. E inspirará en los ciudadanos de todas partes del mundo un sentido más profundo de la responsabilidad que comparten en cuanto a la administración de nuestro pequeño planeta.

Las naciones desarrolladas y en desarrollo, en diversas reuniones internacionales, han llegado a un consenso en el sentido de que la red de información óptima debe construirse sobre cinco principios centrales: inversión privada, competencia, regulación flexible, acceso abierto y servicio universal. El objetivo de estos principios guías es acelerar el desarrollo de la IMI y asegurar su longevidad [...].

Todos esos cinco principios están estrechamente vinculados entre sí y dependen uno del otro para cobrar fuerza. Debemos reflexionar acerca de cómo estos principios pueden adelantar tanto los intereses particulares de las naciones individuales como los intereses comunes de todos los ciudadanos del mundo.

Permítanme examinar los principios centrales.

Comencemos por la inversión privada y la competencia. El presidente Clinton promulgó la Ley de Reforma de las Telecomunicaciones de 1996, la cual abrirá a la competencia de una legión de compañías nuestros mercados de las comunicaciones. Estamos convencidos de que liberar a las empresas privadas para que compitan entre sí ha demostrado, una y otra vez, ser la mejor técnica para encender la creatividad, crear empleos, impulsar los beneficios financieros y llevar a los consumidores toda una gama de nuevos servicios.

Para el sector privado, esta es una oportunidad tremenda -como lo hemos visto en America del Sur, en Asia y ahora en partes de Africa-. Pero la inversión privada, dondequiera que ocurra, debe ir acompañada de una competencia vigorosa.

En los Estados Unidos hemos aprendido esa lección. Cuando un juez federal dividió en varias compañías la American Telegraph \& Telephone (AT\&T), el mayor monopolio telefónico del mundo, los resultados sorprendieron hasta a los más fervientes partidarios de eliminar las regulaciones. El precio de las llamadas telefonicas de larga distancia cayo espectacularmente. Nuevas compañías, con nuevos empleos, aparecieron en escena. La propia AT\&T, finalmente, se convirtió en una compañía más fuerte, más competitiva e innovadora.

Los acontecimientos de Chile ejemplifican también los beneficios de la inversión privada y la competencia abierta. En 1994, Chile estableció una estructura reguladora fuertemente favorable a la competencia.

El nứmero de operadores de langa distancia aumentó en Chile de 1 a 12. La porción de los hogares con servicio telefónico dio un salto de más de un $50 \%$. Y los precios bajaron de alrededor de dos dólares a aproximadamente un quinto de dólar por minuto. También subieron los ingresos de la industria, aproximadamente dos veces más rápidamente que los de la economía en general.

La inversion privada y la competencia son esenciales para el desarrollo de la IMI. También lo es el tercer principio, una regulación inteligente, flexible. Para que los inversionistas corran riesgos y se arraigue la competencia, las regulaciones 
deben asegurar estabilidad, libertad y flexibilidad, en tanto que también les ofrecen a los consumidores precios justos y amplia gama de opciones.

En los Estados Unidos regulamos muchas industrias de las comunicaciones a través de una agencia independiente, la Comisión Federal de Comunicaciones (FCC). Este organismo experto cuenta con la pericia necesaria para tomar decisiones técnicas, y junto con otras agencias de los departamentos de Justicia y Comercio de los Estados Unidos, la FCc dispone de la capacidad para vigilar las condiciones cambiantes del mercado.

En la misma medida en que estas nuevas tecnologías revolucionan el viejo orden comercial, los que estamos en el gobierno debemos echar abajo las estructuras regulatorias obsoletas mientras permanecemos fieles a los valores e ideales subyacentes.

Otro principio central -estrechamente vinculado a los principios de la inversión privada, la competencia y las regulaciones flexibles- es el acceso abierto. Es necesario que todas las naciones y todas las partes puedan conectarse a la imi.

La razón la puede exponer, en parte, un bien conocido principio de la ciencia de la computación, la Ley de Metcalfe. La Ley de Metcalfe sostiene que el poder en una red de computadoras aumenta aproximadamente en proporción directa al cuadrado de la cantidad de personas conectadas con ella.

Ésa es la razón por la que el internet crece con tanta rapidez. Cuanta más gente se conecta, más gente quiere conectarse. Si se duplica la cantidad de gente en línea, se cuadruplica la cantidad de maneras posibles de vincular a esa gente y combinar su talento y sus ideas.

Ésa es la razón por la que el acceso abierto es tan importante. Manténgase a la gente fuera de la red, y la red no será tan valiosa. Dejese entrar a la gente, y el valor que cada uno obtiene se disparará.

Por lo tanto, los dueños de las redes deben cobrar precios de acceso a las mismas que no sean discriminatorios. La única manera de materializar la promesa verdadera de la IMl garantizar que cada uno de los que se conectan tendrá acceso a miles de fuentes de información diferentes - descle la programación de videos a los periódicos electrónicos y los tablones de anuncios computarizados- de cada nación, en cada idioma.

El quinto y último principio es, tal vez, el más importante: el servicio universal. Estamos convencidos de que el servicio universal puede ser un resultado natural de los primeros cuatro principios. Por cierto que la combinación de acceso abierto, regulaciones flexibles, competencia e inversion privada nos arrastrará en esa dirección. Pero, por sí mismos, no nos llevarán de lleno hacia ese destino.

Ésa es la razón por la que el presidente Clinton y yo hemos retado al sector privado de nuestra nación a ayudar a conectar cada escuela de Norteamérica a la supercarretera de la información para fines de este siglo. Y ésa es la razón por la que reitero mi llamado a la creación de una Biblioteca Digital Mundial, para que todos los ciudadanos del mundo tengan acceso más rápido y más rico a toda la información del mundo.

Por supuesto, en cada nación diferirán los contornos exactos del servicio 
universal. Pero su forma fundamental debe ser similar en la mayor parte de las localidades. Por ejemplo, proveer servicios básicos a precios accesibles a la gente de todos los niveles de ingresos, hacer que esté disponible servicio de alta calidad, no importa cuál sea la situación geográfica o capacidad física de una persona, y enseñaries a los consumidores cómo usar con efectividad estas tecnologías.

La IMI es una iniciativa histórica. La fortalece la participación, la sostiene la apertura, $y$ la fortifican las naciones vigorosas y la gente talentosa que van en pos de sus ideales de un mañana mejor.

Únanse a mí en la construcción del primer gran logro del siglo xxa2.

El 1 de julio de 1997, el gobierno de los Estados Unidos promovió la iniciativa "Framework For Global Electronic Commerce". En ese documento, Gore sintetizó sus principales tesis sobre el desarrollo la sociedad de la información:

1. The private sector should lead (el sector privado debe liderar).

Internet debe desarrollarse como un terreno competitivo, regulado por el mercado y no por el gobierno. Aun cuando se requiera la intervención estatal, los gobiernos deben propiciar que sea la industria la que se autorregule, y que sea el sector privado el líder, siempre que esto sea posible.

2. Governments should avoid undue restrictions on electronic commerce (los gobiernos deben evitar restricciones al comercio electrónico).

Los gobiernos deben frenar su inclinación a imponer nuevas e innecesarias regulaciones, procedimientos burocrâticos, nuevos impuestos o aranceles a las actividades en la Web.

3. Where governmental involvement is needed, its aim should be to support and enforce a predictable, minimalist, consistent and simple legal environment for commerce (cuando la intervención gubernamental sea requerida, ésta debe destinarse a propiciar que el comercio tenga un entorno jurídico simple, predecible y coherente para estimular un ambiente propicio para el comercio).

El gobierno no debe regular internet. El gobierno debe limitarse a estimular condiciones de competencia, proteger la privacidad y la propiedad intelectual, prevenir el desarrollo de prácticas fraudulentas, asegurar condiciones de transparencia y facilitar la solución de posibles conflictos.

4. Governments should recognize the unique qualities of the internet (los gobiernos deben reconocer las caracteristicas únicas de internet).

El éxito sin precedentes de internet, puede atribuirse a su naturaleza descentralizada, y a su forma de gobierno, la cual es relativamente plana. Ias normas que se crearon en los últimos sesenta años para regular las telecomunicaciones, la radio y la televisión, no se ajustan a internet. Las normas actuales que puedan perjudicar el desarrollo del comercio electrónico deben ser revisadas, eliminadas o ajustadas a las necesidades de la nueva era electrónica. 
5. Electronic Commerce over the internet should be facilitated on a global basis (el comercio electrónico debe ser facilitado mundialmente).

Internet es un terreno competitivo mundial. La estructura jurídica que sustente las transacciones comerciales por internet debe ser consistente y predecible, independientemente del domicilio de quien compre o de quien venda.

El 12 de octubre de 1998, en el discurso que Al Gore pronuncio ante los agremiados al Sindicato Internacional de Telecomunicaciones, el entonces vicepresidente de los Estados Unidos expuso las cinco tesis centrales de "La declaración de la Independencia Digital". Éstas son:

1. Debemos mejorar el acceso a la tecnologia, a fin de que cada uno de los individuos en el planeta cuente, a corto plazo, con los servicios de telecomunicaciones de voz y datos en el transcurso de la siguiente década.

2. Debemos superar nuestras barreras de lenguaje y desarrollar tecnología con traducción digital en tiempo real, a fin de que cualquier individuo del planeta pueda hablar con otro en cualquier lugar del mundo.

3. Debemos crear una Red Global del Conocimiento con la gente que está trabajando para cumplir con nuestros desafios más importantes en materia de educación, cuidado de la salud, recursos agrícolas, desarrollo sostenido y seguridad pública.

4. Nosotros debemos usar la tecnología de las comunicaciones para asegurar el libre flujo de ideas y para apoyar la democracia y el libre discurso.

5. Nosotros debemos usar las tecnologías de la comunicación para realizar la expansión de oportunidades económicas a rodas las familias y a todas las comunidades en el mundo [Leer, 2001: 25].

Es posible afirmar que las tesis de $\mathrm{Al}$ Gore fueron indispensable referente conceptual en el desarrollo de la inmensa mayoría de programas de eGobierno en el mundo. El principal postulado de Gore, definitivamente resulta elocuente: "La gente en el primer plano de la era de la información".

\section{El Sistema Nacional e-México y la reforma de la administración pública}

Hacia la media tarde del domingo 2 de julio de 2000 , los resultados de las primeras encuestas de salida permitian anticipar el triunfo de Vicente Fox Quesada, candidato a la Presidencia de la República de la "Alianza por el cambio", coalición que formaron el Partido Acción Nacional (PAN), y el Partido Verde Ecologista de México (PVEM). Ante la inminente derrota, algunos asesores de Francisco Labastida Ochoa, candidato del Partido Revolucionario Institucional (PRI), y ciertos líderes de los sectores "duros" del prísmo histórico, de nueva cuenta parecían dispuestos a cometer un 
escandaloșo "fraude patriótico" que permitiría imponer al candidato presidencial del partido tricolor. De ese modo, el PRI conservaría el poder. El doctor Ernesto Zedillo Ponce de León, entonces presidente de México, paró en seco a los apologetas de la democracia priista, y pocos minutos después de las 11 de la noche, a través de la radio y televisión en cadena nacional, procedió a reconocer el triunfo de Vicente Fox.

En su campaña en pos de la Presidencia de la República, Vicente Fox prometió hasta lo imposible. Fox se presentó a sí mismo, no al PAN, como esperanza y respuesta a cada uno de los graves problemas de nuestro México. Gracias a un efectivo trabajo de mercadotecnia política, el "producto Vicente Fox" consiguió sintetizar las esperanzas ciudadanas de dar fin a los excesos de la llamada "presidencia imperial", e inaugurar los días de un nuevo tiempo mexicano.

El Sistema Nacional e-México forma parte de las iniciativas que emprendió el gobierno del presidente Vicente Fox para impulsar la indispensable transformación de las instituciones públicas. El Programa Nacional e-México dio formal inicio el 22 de febrero de 2001 , en Pueblo Nuevo, Durango. Ese día, el presidente Fox inauguró el telecentro de El Salto, Durango. De acuerdo con el doctor Julio César Margáin y Compean, entonces director del Sistema Nacional e-México:

[...] el Sistema e-México es un proyecto integrador que busca articular los intereses de los distintos niveles de gobierno, de diversas entidades y dependencias públicas, de los operadores de las redes de telecomunicaciones, así como de muchas instituciones públicas y privadas, a fin de ampliar la cobertura de servicios de salud, educación, comercio y gobiemo, así como de otros servicios a la comunidad [Margáin, 2001: 3].

El presidente Vicente Fox decidió delegar en la Secretaría de Comunicaciones y Transportes (ScT), la responsabilidad de coordinar el desarrollo del Sistema Nacional e-México, el cual comprendía cuatro subsistemas: tecnologias e interconexión; contenidos y programas; marco legal y tarifario; administración y gestión. De acuerdo con el doctor Margáin, los objetivos del Programa Nacional e-México eran los siguientes:

[...] reducir la brecha digital entre México y el mundo; entre el gobierno, las empresas, las familias; incrementar la penetración y cobertura de servicios como telefonía e internet, así como servicios afines; eliminar barreras de acceso a la información, el conocimiento, los servicios y los mercados; facilitar el acceso a diversos servicios como salud, educación, comencio, y los servicios y trámites del gobierno; incorporar a las pequeñas y medianas empresas, así como a los microproductores antesanales y de diversas actividades regionales en la denominada nueva economía; eliminar cadenas de intermediarios que no agreguen valor a las actividades productivas y comerciales de las empresas; difundir nuestra riqueza 
pluricultural, asi como los atractivos naturales y turísticos que México ofrece al mundo [Margáin, 2002: 11].

Uno de los principales objetivos de la primera etapa de desarrollo del Sistema Nacional e-México, consistía en integrar los esfuerzos de diversas dependencias e instituciones públicas y privadas para generar servicios y contenidos en línea, indispensables para el desarrollo de los programas de e-Gobierno, e-Educación, e-Salud, y e-Comercio. De acuerdo con el doctor Margáin, e-Gobierno:

[...] es un medio para que todos los mexicanos, en el ámbito federal, regional, estatal y municipal, puedan ejercer su derecho a estar informados y acceder a los servicios que ofrece el Estado, a través de la Mega Red dèl Sistema e-México. Asimismo, el Estado, a través de las diferentes instancias del gobierno, asume la obligación de garantizar el acceso de toda la población a la información, uso y aprovechamiento de los diversos servicios públicos que ofrece. Dentro de esos servicios la población podrú realizar consultas y trámites a través de la Mega Red, como el acceso a información con respecto al ejercicio del Presupuesto de Egresos de la Federación, realizar trámites como la declaración de impuestos, solicitud de permisos y licencias, pasaportes y cualquier trámite, de tal manera que la terminal, el quiosco o una computadora, se convierta en una ventanilla de atención a ciudadanos, transparentando y agilizando los servicios [Margáin, 2002: 12].

Para Abraham Sotelo Nava, titular de la Unidad de Gobierno Electrónico y Política de Tecnologías de la Información de la Secretaría de la Función Pública, y Líder de la Estrategia de Gobierno Digital, sub-Sistema del Sistema Nacional e-México, e-Gobierno es una estrategia vertical dentro de la iniciativa e-México, la cual:

[... persigue el propósito de modificar radicalmente la forma como el gobierno se comunica con la ciudadanía y presta servicios para satisfacer necesidades públicas [...] es la innovación continua de los servicios, la participación de los ciudadanos y la forma dè gobernar mediante la transformación de las relaciones externas $\mathrm{e}$ internas a través de la tecnología, el internet y los nuevos medios de comunicación. En otras palabras, el e-Gobierno implica una transformación de la función gubernamental introduciendo nuevos valores de comunicación, cooperación y participación interactiva para la toma de decisiones gubernamentales ( $\mathrm{g} 2 \mathrm{c}$ : Relaciones gobiemociudadano). De igual manera se modificarán las estrategias de transparencia, desempeño y rendición de cuentas al interior del gobierno ( $\mathrm{g} 2 \mathrm{~g}$ : Relaciones gobiemogobierno) para que éste actúe más eficientemente en la presentación de los servicios y en la administración de la información, reforzando su liderazgo frente a la sociedad. Finalmente, las relaciones gobierno-mpresas ( $\mathrm{g} 2 \mathrm{~b}$ government-business) serán también rediseñadas buscando la participación activa y comprometida del sector privado en la modernización gubernamental [Sorelo, 2002: 15]. 
Abraham Sotelo Nava subrayó que la innovación del gobierno representa un profundo movimiento culnural y estructural, el cual persigue el propósito de reformar radicalmente la orientación, capacidad y velocidad de respuesta de la administración pública federal frente a asuntos que admiten un carácter estratégico. De acuerdo con Sotelo Nava, las principales aplicaciones genéricas que podrían derivarse del desarrollo del "gobierno electrónico" son:

[...] control del tránsito; e-taxation (cobro de impuestos); pago de servicios (uso de técnicas del comercio electrónico); voto electrónico; seguridad pública (prevención y respuesta ante accidentes); buzón de denuncias; ámbitos de comunicación directa en tiempo real de funcionarios en audiencias públicas ( $c b a t s$ ); encuestas; licitaciones públicas; tramitación y renovación de permisos y licencias; información sobre servicios de la administración pública; guía de trámites; bolsa de trabajo; registro de solicitudes de beneficios sociales diversos (Progresa, Fonaes); iniciativa popular (ámbito para propuestas de legislación o normas diversas por parte del público); servicios para sectores segmentados (jóvenes, mujeres, jubilados); registro de agenda y eventos; y, en general, poder involucrar a la ciudadanía en el proceso de deliberación previo a la toma de decisiones [Sotelo, 2002: 15].

Raúl Méndez Segura, entonces director general y socio responsable en México de la incubadora de internet de Bozz, Allen \& Hamilton, advertía que el reto más delicado del Sistema e-México era la "brecha digital"; es decir, la división que existe entre los que tienen acceso a las avanzadas tecnologias de información y comunicaciones, incluyendo internet, y quienes no. Raúl Méndez destacó que las graves insuficiencias de nuestra infraestructura de telecomunicaciones representan un difícil obstáculo al desarrollo del Sistema e-México. En el año 2001, México presentaba una de las teledensidades más bajas en América Latina (11.9 líneas telefónicas por cada 100 habitantes), cifra inferior a países como Panamá (16.5), Brasil (14.9), y Costa Rica (20.4). La penetración de computadoras en México (6.3 computadoras por cada 100 habitantes), además era inferior a Belice (10) y Unuguay (11.4). Para comprender la magnitud del esfuerzo que debian realizar las instituciones gubernamentales para poner en marcha el Sistema Nacional e-México, resultaba indispensable evaluar el nivel de coordinación e integración de los proyectos que estaban ejecutando las instituciones gubernamentales a nivel federal, estatal y municipal en materia de internet; el grado de desarrollo adicional que podrían requerir los procesos internos clave para responder a la oferta de servicios potenciales en internet; el grado de desarrollo de la normatividad informática y de internet aplicable a lo largo de las distintas entidades gubernamentales; el grado de eficiencia con el cual las diferentes dependencias gubernamentales comparten información relevante; y la magnitud de las diferencias de tecnología y capacitación entre las distintas entidades. 
Abraham Sotelo Nava efectivamente reconocía las graves dificultades que podría enfrentar el desarrollo del Sistema Nacional e-México. En una entrevista que concedió a la periodista Mónica Mistretta, directora de la prestigiada revista Information Week, Sotelo Nava describió el caótico panorama que presentaba la infraestructura tecnológica de la administración pública al inicio del gobierno del presidente Vicente Fox: "300 ERP distintos instalados, 15,000 aplicaciones diferentes, 45,000 programadores y analistas (para darse una idea, Microsoft tiene 9,000 en todo el mundo) y $83 \%$ del presupuesto destinado a mantener las aplicaciones instaladas (Mistretta, 2002: 12).

El 1 de agosto de 2001, en la columna "Templo Mayor" del periódico Reforma, F. Bartolomé realizó un duro cuestionamiento al Sistema Nacional e-México:

A casi cinco meses de que se realizaron los foros de consulta sobre el programa eMéxico, ni quien se acuerde de lo que se suponía iba a ser una gran red para celebrar transacciones con el nuevo gobierno. Las ponencias ahí están, en internet como debe de ser, pero la Secretaría de Comunicaciones y Transportes de Pedro Cerisola nomás no da luces sobre el tema. Lo preocupante del asunto es que, aun y cuando se supone que e-México sería algo así como la puerta de entrada del gobierno a la modemidad, la realidad indica que los foxistas no han encontrado al cerrajero que la abra. Porque una cosa es pretender ofrecer trámites, acceso a la información, educación y hasta servicios de salud a través de internet y otra muy diferente que la red de redes sólo sirva para ser otra vía de difusión de las labores gubernamentales que actualmente ya se hace, hasta el cansancio. Todavía están a tiempo los muchachos de Pedro Cerisola de no cometer el mismo error que la Contraloría con ese ciberelefante blanco llamado Compranet. O sea, basta de ciber rollos [Bartolomé, 2001].

En septiembre de 2002, poco después de la presentación del Primer Informe e-Gobiemo, Abraham Sotelo Nava denunció el tipo de resistencias que enfrentaba el programa de modernización de la administración pública propuesto por el presidente Vicente Fox. "Del $100 \%$ de personas al interior del gobierno, $10 \%$ estamos dedicados a tratar de transformarlo, $80 \%$ paga por ver y otro $10 \%$ en posiciones medias o superiores se vuelven obstaculizadores y hasta practican una especie de sabotaje" (Mistretta. 2002: 15). De acuerdo con Abraham Sotelo, los principales obstáculos al cambio estructural de la administración pública eran: resistencia a la rendición de cuentas, inercias culturales negativas, abuso de poder por parte de los servidores públicos, falta de capacidades técnicas y financietas, desconexion entre los valores inculcados a los funcionarios y sus acciones, desprecio hacia la innovación como habilidad organizacional. 
Las fricciones entre los grupos de poder incrustados en el gobierno del presidente Vicente Fox alcanzan al Sistema Nacional e-México

Para colmo, algunos funcionarios de primer nivel del gobierno del presidente Vicente Fox, e ilustres figuras del "panismo doctrinario", empezaron a ventilar sus diferencias en los medios de comunicación. El gabinete del presidente Vicente Fox distaba mucho de ser un equipo efectivamente disciplinado. Miembros de algunos de los grupos en confrontación, por ejemplo, el "sector aristocrático" del Partido Acción Nacional, empezaron a objetar el desempeño de algunos de los altos funcionarios del gobierno del presidente Vicente Fox. En el semanario Proceso, Felipe Calderón Hinojosa enjuició con sorprendente severidad el desempeño de Santiago Creel, titular de la Secretaría de Gobernación. La diputada Teresa Gómez Mont realizó enérgicos cuestionamientos al desempeño de la señora Marta Sahagún Jiménez, responsable de la Dirección General de Comunicación Social de la Presidencia de la República, antes de convertirse en esposa del presidente Vicente Fox, el 2 de julio de 2001. No pocos dirigentes del "panismo doctrinario" todavía consideraban a Vicente Fox como el oportunista que había utilizado al PAN para ascender a la Presidencia de la República.

El enfrentamiento entre los grupos que ambicionaban incrementar sus cuotas de poder al interior del gobierno del presidente Vicente Fox, pronto trascendió al escenario del Sistema Nacional e-México. En el texto "Sistema de Trámites Electrónicos Gubernamentales", el cual fue publicado en el número 2 del Boletín de Política Informática, se afirma lo siguiente:

[...] el gobierno federal puso en marcha el Sistema de Trámites Electrónicos Gubernamentales (Tramitanet), cuyo objetivo es proporcionar a la ciudadanía mecanismos ágiles de consulta de información sobre todos los trámites gubernamentales, asî́ como para realizartos en forma electrónica [...] Tramitanet, uno de los componentes fundamentales del Programa de Gobiemo Electronico, diseñado por la Secretaría de Contraloría y Desarrollo Administrativo (Secodam) en coordinación con todas las dependencias y entidades de la Administración Pública Federal (APF), representa un esfièzo de transparencia y desregulación en la gestión gubernamental, para inhibir actos de corrupción y discrecionalidad entre los servidores públicos y conto con la participación inicial de la Secretaría de Gobemación (Segob), la Secretaría de Economía (SE), la Secretaría de Educación Pública (SEP), y el Instituto Mexicano del Seguro Social (IMss)" [Boletín de Politica Informática, 2002: 1].

Las omisiones que es posible inferir en la referida gacetilla, exhiben el fuego "poco amistoso" que ya sostenían ilustres miembros del "panismo doctrinario", con determinados funcionarios del gobierno de Vicente Fox. 
En primer lugar, en el referido texto no fue mencionada la existencia del Sistema Nacional e-México; en segundo lugar, no se hizo mencion alguna al hecho de que el presidente Vicente Fox delegara en la Secretaría de Comunicaciones y Transportes (SCT), el desarrollo del Sistema Nacional eMéxico; en tercer lugar, fue ignorado el "Programa e-Gobierno", a cargo de Abraham Sotelo Nava.

El "Programa del Gobierno Electrónico", diseñado por la Secretaría de Contraloria y Desarrollo Administrativo (Secodam), cuyo titular era Francisco Barrio, duplicaba algunos de los objetivos del Sistema Nacional eMéxico, particularmente los comprendidos en el "Programa e-Gobierno de la Presidencia para la innovación gubernamental". ¿Acaso el presidente de la República, como los titulares de Secodam y de SCT, no advirtieron que la iniciativa presentada por Secodam representaba un acto de "piratería cibernética"? Si efectivamente el gobierno federal disponía de limitados recursos para impulsar el desarrollo del Sistema Nacional e-México, eno convendría haber subordinado el Programa de Gobierno Electrónico de la Secodam, al Programa e-Gobierno de la Presidencia de la Oficina para la Innovación Gubernamental, el cual efectivamente formaba parte del Sistema Nacional e-México, evitando así la duplicación de esfuerzos?

El entonces titular de la Secodam, Francisco Barrio Terrazas, fue gobernador del estado de Chihuahua durante el gobierno del presidente Carlos Salinas de Gortari. En la actualidad, Francisco Barrio es identificado como uno de los principales dirigentes del PaN y se desempeña como coordinador de la bancada blanquiazul en la Cámara de Diputados. En su gestión como titular de Secodam, Francisco Barrio arrojó pobres resultados, particularmente en la lucha contra la corrupción. Barrio prometió capturar "peces gordos" y no cumpli6; sus inoportunas declaraciones generaron un delicado enfrentamiento con el secretario de Hacienda. En otra ocasión, sus torpes declaraciones generaron un profundo malestar entre altos jerarcas de la Iglesia católica. En alguna comparencia en la Cámara de Diputados, Barrio reconoció que fueron utilizados recursos públicos del Ramo 33, por la cantidad de diez millones de pesos, para la remodelación del rancho San Cristóbal, propiedad del presidente Vicente Fox, con motivo de la visita de George W. Bush, presidente de los Estados Unidos, en febrero de 2001. Francisco Barrio frecuentemente filtró a la prensa información relacionada con averiguaciones emprendidas por la Procuraduría General de la República (PGR) contra presuntos responsables de actos de corrupción, quienes, en su mayoría, consiguieron evadir la justicia. El cúmulo de errores de Francisco Barrio terminó por incidir en el ánimo del presidente Vicente Fox, quien decidió apartarlo de su gabinete y apresurar el cambio de nombre de la Secodam, la cual fue convertida en la "Secretaría de la Función Pública".

Pedro Cerisola y Weber, titular de la Secretaría de Comunicaciones y 
Transportes (SCT), ingresó a "Los Amigos de Fox" el 15 de septiembre de 1999 y de inmediato se convirtió en uno de los selectos miembros del "primer círculo" de la campaña presidencial del candidato de la "Alianza por el Cambio". Antes de incorporarse al equipo de campaña de Vicente Fox, Cerisola se desempeñó como director de AeroMéxico, y también ocupó algunos cargos directivos en Telmex. Carlos Slim, propietario de Telmex, y el hombre más rico de América Latina, precisamente recomendó a Pedro Cerisola con Vicente Fox. Pedro Cerisola realizó los trámites de su titulación profesional, en la UNAM, poco antes de ser designado secretario de Comunicaciones y Transportes. Además gestionó su ingreso al PAN ya como titular de la sCT.

\section{Relaciones peligrosas. El amigo Bill Gates}

En la entrevista que concedió Bill Gates a la reportera Claudia Olguín, la cual fue publicada en Milenio Semanal, el dueño de la mayor fortuna privada en el orbe, estimada en más de 50 mil millones de dólares, declaró:

Microsoft y la Fundación Gates hicieron algunos donativos al gobiemo mexicano, de computadoras personales, que estarán en bibliotecas públicas. La iniciativa fue parte de un anuncio conjunto con el presidente Fox, su esposa y Melinda. Significa que cualquier niño que tenga acceso a una biblioteca puede tener acceso a una computadora. Esto ha sido muy exitoso en los Estados Unidos y hace sentido en otros paises. Si se analiza el trabajo de los negocios más importantes en México, están usando el mismo camino que los Estados Unidos, donde se considera que la comunicación debe ser eficiente [Olguín, 2002: 38].

El miércoles 17 de abril de 2001, en Seattle, Estados Unidos, el gobierno de México y Microsoft firmaron un convenio para instalar 2,473 centros comunitarios digitales, los cuales, así fue informado, formarían parte del Sistema Nacional e-México. Pedro Cerisola, titular de SCT, y Rick Belluzzo, presidente de Microsoft, firmaron el referido convenio en el marco de la "V Conferencia de Líderes Gubernamentales", evento organizado por Microsoft, al cual el presidente Vicente Fox finalmente no pudo asistir, pues el Senado de la República no le concedió autorización para viajar a los Estados Unidos. El presidente Fox, sin embargo, dictó una conferencia a través de un videoenlace. El doctor Julio César Margáin y Compean, entonces director del Sistema Nacional e-México, afirm6 que el convenio que suscribió el gobierno del presidente Vicente Fox con Microsoft: "se inscribe dentro del ambicioso y amplio proyecto de eMéxico con el que se busca colocar a la nación mexicana dentro de las sociedades informáticas del mundo". 
A pesar de los propósitos de transparencia que insistentemente ha pregonado el presidente Vicente Fox, el convenio que firmaron Pedro Cerisola, titular de SCT, y Rick Belluzzo, director de Microsoft, nunca fue publicado en el sitio Web del Sistema Nacional e-México. Toda la información relativa al citado convenio que era posible consultar en las páginas WwW dẹl Sistema Nacional e-México (octubre de 2002), se limitaba a los siguientes párrafos:

"México y Microsoft concretaron hoy un convenio de colaboración para desarrollar 2,473 centros comunitarios digitales, que integrarán el proyecto e-México, con el que se busca dar acceso a internet a todo el país en 2006. El convenio fue firmado este miércoles en una reunión entre el secretario mexicano de Comunicaciones, Pedro Cerisola, y el presidente de Microsoft, Rick Bèlluzzo, en el marco del último día de la $\mathrm{V}$ Conferencia de Líderes Gubernamentales, que organiza la empresa. En el acuerdo, la compañía tecnológica -con sede en Seattle- se compromete a proporcionar gratuitamente el $10 \%$ de programas de computación (software) que utilicen las computadoras que sean instaladas en los Centros Comunitarios Digitales ( $C \mathrm{CD}$ ). Microsoft se compromete además a vender a precios bajo el resto de los programas y aplicaciones que el gobiemo mexicano le solicite para dichos centros. La compañía tecnológica también se compromete a capacitar en el uso de sus productos a unos 4,500 operadores de los CCD de manera gratuita. La capacitación se efectuaría en el transcurso de este año en 10 puntos del país. El gobierno mexicano pretende como parte del proyecto e-México establecer durante el año 2002, al menos un CCD en cada una de las cabeceras municipales del país, por más pequeñas y remotas que sean. La meta es, eventualmente, expandir el número de $C C D$ o de kioskos de enlace a internet a unos 10 mil para el año 2006. LOS CCD brindarán a los ciudadanos acceso a internet y servirán también como puntos de entrenamiento y capacitación en el uso de las nuevas tecnologías. El convenio fue furmado por Julio César Margáin, coordinador del proyecto e-México, y Felipe Sánchez, director de Microsoft México, y atestiguado por Cerisola y Belluzzo. Tras la firma, Margáin dijo que este convenio se inscribe dentro del ambicioso y amplio proyecto de e-México con el que se busca colocar a la nación mexicana dentro de las sociedades informáticas del mundo. Sánchez señaló, por su parte, que la colaboración con las autoridades mexicanas forma parte del compromiso de Microsoft por promover el uso de las nuevas tecnologías en México. Lo que boy pusimos en la mesa tiene que ver con conectitidad, explicó Sánchez, al destacar la importancia de la creación de los CCD para llevar el acceso de la internet a todos los rincones de México. El secretario Cerisola dijo en su oportunidad que Microsoft es una de las múltiples compañías privadas, instituciones ciudadanas y organizaciones ciudadanas que participan dentro del proyecto e-México. El funcionario respondio asi a las críticas que algunos programadores e ingenieros en informática han formulado al hecho de que el gobiemo utilice software de compañías como Microsoft, en lugar del que existe disponible de manera libre en forma gratuita. "e-México es un proyecto de arquitectura abierta", 
explicó Cerisola, al asegurar que en el mismo se utilizarán todas las herramientas tecnológicas que le sirvan. El convenio firmado aquí es adicional a uno más grande que se anunciará próximamente entre Microsoft y el gobierno mexicano y bajo el cual la compañía tecnológica contribuirá con una inversión de 58 millones de dolares para promover la industria del software en México. Funcionarios mexicanos y ejecutivos de Microsoft han estado trabajando durante el último año en el proyecto, luego de la entrevista que el presidente de México Vicente Fox, sostuvo con Bill Gates, fundador de la compañía. Sánchez adelantó a Notimex que en el plan a anunciarse en las proximas semanas, Microsoft planea capacitar en la creación y manejo de software a unos 20 mil técnicos certificados y a unos tres mil profesores. El plan formaría parte del primer programa gubernamental mexicano para promover el establecimiento y desarrollo de la industria del sector informático en México".

¿Cómo no sospechar de las relaciones de negocios que ha fincado Microsoft con el gobiemo del presidente Vicente Fox, si el referido convenio no se dio a conocer a la opinión pública? ¿Por qué no fue dado a conocer? ¿Qué tan desinteresado puede ser el altruismo que ha exhibido Bill Gates hacia el gobiemo de Vicente Fox? ¿Por qué? ¿Acaso Microsoft forma parte de las empresas que realizaron aportaciones irregulares a la campaña presidencial de Fox, a través de "Los Amigos de Vicente Fox" mediante algún otro fideicomiso capaz de triangular grandes cantidades de dinero, como "Desarrollo y Democracia", por ejemplo? ¿Cuántas donaciones ha realizado la Fundación Bill \& Melinda Gates a la "Fundación"Vamos México"? ¿A cuánto ascienden esas donaciones? ¿Qué interés tiene Microsoft en el Sistema Nacional e-México para mantener en su sitio Web un apartado dedicado al tema de e-México? ¿Por qué Steve Ballmer cometió la indiscreción de anticipar que Microsoft crearía el portal gubernamental de México? ¿A qué portal se refería? ¿Por qué ante las imprudentes declaraciones de Ballmer, los funcionarios responsables del Sistema Nacional e-México se limitaron a guardar silencio?

Muchas de las acciones filantrópicas de la Fundación Bill \& Melinda Gates, observan una estrecha y evidente relación con los intereses comerciales de Microsoft. Ello permite poner en duda la autenticidad de las generosas acciones de filantropía que tanto publicita la Fundación Gates, particularmente en aquellos países en los cuales son consideradas soluciones de software abierto, o Free/Libre and Open-Source Software (FLOSs), para el desarrollo de programas de E-gobierno. En India, por ejemplo, poco después de que el Departamento de Información anunció que todos los servicios en línea de instituciones educativas y gubernamentales correrían a través de plataformas Linux, Bill Gates dio a conocer que la Fundación Bill \& Melinda Gates donaría una considerable cantidad de recursos para combatir la propagación del viH en la India: 
La Fundación Bill y Melinda Gates está en India esta semana para establecer un compromiso a largo plazo con sus socios indios acerca de una nueva e importante iniciativa de prevención dirigida a las llamadas poblaciones moviles que incluyen chóferes de camiones, soldados y trabajadores migrantes. La iniciativa se centrará en estrategias de prevención probadas, como asesoria voluntaria y distribución de condones, además del tratamiento de enfermedades de transmisión sexual y programas de educación para reducir temores y el estigma que se relaciona con el sida [Gates, 2002: 2].

Gates ni siquiera disimuló la importancia que representa la India para los intereses comerciales de Microsoft:

India está en camino de convertirse en una superpotencia económica global. Su economía ha superado al resto de Asia en los últimos años; se vaticina que sus sectores de tecnología de la información y farmacéutico, competitivos a nivel internacional, crecerán dramáticamente esta década, y el poder de compra del país es ahora el cuarto del mundo después de los Estados Unidos, China y Japón. [...] Con los años, he desarrollado lazos profesionales y personales estrechos con India. Su industria del software - de gran crecimiento- ha hecho del país un socio crucial de muchas empresas estadounidenses, entre ellas, Microsoft [Gates, 2002: 2].

Muchos especialistas afirman que el software abierto representa una atractiva alternativa frente al costoso software propietario de Microsoft, cuyas licencias se convierten en permanente hipoteca. El software abierto permite que los interesados en el desarrollo de determinadas plataformas, a través de internet puedan tener acceso a los códigos de los programas, situación que facilita identificar rápidamente posibles errores, e incorporar las mejorias pertinentes a partir de extensiones. Linux no solo ha configurado el mercado y ha estimulado la progresiva demanda de programas de código abierto. El desarrollo de soluciones en ambiente Linux definitivamente estorba a Bill Gates, quien se encuentra dispuesto a convertir a la "Sociedad de la Información y el Conocimiento" en el mejor negocio de Microsoft. Microsoft de ninguna manera parece dispuesta a abrir los codigos de su software, el cual pretende imponer a cada uno de los gobiernos que se encuentran considerando soluciones en materia de e-Gobierno.

En muchos países - México de ninguna manera es la excepción-, impulsores de soluciones de software libre han insistido en la necesidad de incorporar leyes que permitan regular asuntos relativos al software a emplear por el Estado. En Perú, por ejemplo, algunos legisladores han elaborado determinadas iniciativas de ley en la materia. En el proyecto de ley número 1609, denominado "Software Libre en la Administración Pública", se asienta lo siguiente: 
El software, para ser aceptable para el Estado, no basta con que sea técnicamente suficiente para llevar a cabo una tarea, sino que además las condiciones de contratación deben satisfacer una serie de requisitos en materia de licencia, sin los cuales el Estado no puede garantizar al ciudadano el procesamiento adecuado de sus datos, velando por su integridad, confidencialidad y accesibilidad a lo largo del tiempo.

En la exposición del Proyecto de Ley Número 1609, relativo al empleo de software libre en la administración pública, el cual fue presentado el 14 de diciembre de 2001 al Congreso de Perú, fueron incorporados los siguientes argumentos para fundamentar la pertinencia de adoptar soluciones de software abierto para el desarrollo de los programas de gobierno electrónico de ese país:

Un gobierno debe garantizar que los ciudadanos tengan libre acceso a la informa. ción del gobierno: para lograr esto, es necesario que la codificación de los datos no se encuentre ligada a un solo proveedor. El uso de formatos abiertos y estándar garantiza este libre acceso, haciendo posible la creación de software compatible.

Un gobierno debe garantizar que la información priblica estê permanentemente dispontble: resulta necesario que el uso y mantenimiento del software no dependan de la buena voluntad de los proveedores, ni de condiciones monopólicas impuestas por ellos. La disponibilidad permanente de la información pública sólo puede garantizarse por la disponibilidad del código fuente del software usado par̆a acceder a esa información.

Un gobierno debe garantizar la seguridad nacional: resulta indispensable tener sistemas libres de elementos que permitan control remoto o la transmision secreta de datos a terceros. Por ello, es imprescindible tener sistemas cuyo código fuente sea libremente accesible al público, para que su inspección por el Estado, los ciudadanos y un gran número de expertos independientes sea posible.

Juan Alberto González, gerente de Microsoft en Perú, emprendió un enérgico cuestionamiento al Proyecto de Ley Número 1609, "Software Libre en la Administración Pública", por considerario contrario al espíritu de "libre empresa". Edgar David Villanueva Núñez, legislador peruano, respondió de la siguiente manera al gerente de Microsoft en Perú:

Para garantizar la seguridad del Estado o seguridad nacional, resulta indispensable contar con sistemas desprovistos de elementos que permitan el control a distancia o la transmisión no deseada de información a terceros. Por lo tanto, se requieren sistemas cuyo código fuente sea libremente accesible al público para permitir su examen por el propio Estado, los ciudadanos, y un gran número de expertos independientes en el mundo. 
Como las soluciones en materia de e-Gobierno y e-Educación representan $20 \%$ de los ingresos de Microsoft en Iberoamérica, la Fundación Bill \& Melinda Gates enseguida anunció la intención de realizar importantes donaciones al sector educativo de. Perú. Podemos concluir que las donaciones que realiza la Fundación Bill \& Melinda Gates, de ninguna manera resultan genuinas y desinteresadas y que, en realidad, representan una especie de sobornos a aquellos gobiernos en los cuales Microsoft siente amenazados sus intereses.

Respecto al espíritu de "libre empresa" que tanto preocupa al gerente de Microsoft en Perú, vale la pena señalar que en junio de 2004, Microsoft fue multada por la Unión Europea con 497.2 millones de euros por "abuso de posición dominante". También, durante la administración del presidente William Clinton, el gobierno de los Estados Unidos demandó a Microsoft por prácticas monopólicas.

Si bien el monto de la referida multa resulta una suma ridícula para Microsoft, el efecto "simbólico" de la misma debe representar motivo de preocupación para Bill Gates. La Unión Europea desea que Microsoft comercialice por separado el sistema operativo Windows y el reproductor multimedia Media Player. Además, ha ordenado a Microsoft compartir ciertos códigos de programación de Windows, "para garantizar la compatibilidad con servidores ajenos a Microsoft". Sin reparar siquiera en el elemental cuidado de las formas, la defensa de Microsoft corrio a cargo del actual responsable de la política antimonopolio de los Estados Unidos -el fiscal general adjunto Hewitt Pate-, quien se mostró "preocupado" "por las posibles implicaciones de la reciente decisión contra Microsoft por abuso de posición dominante. Microsoft recurrirá al Tribunal de la Unión Europea para iniciar las apelaciones correspondientes".

Con el propósito de atajar el desarrollo de soluciones de software abierto, impulsores de la llamada "Iniciativa por la Opción en Software" (Iniciative for Software Cboice), afirman que las leyes que promueven el uso de software de código abierto para soluciones en materia de gobierno electrónico, resultan anticompetitivas y perjudiciales para el comercio. Entre las compañias que participan en la iniciativa, destacan Intel (Estados Unidos), Open Solutions (Argentina), Paradigma (Brasil), Asociación Peruana de Software (Perú), Siam Commercial Bank (Tailandia), vsi (Alemania) $y$, por supuesto Microsoft (Estados Unidos). Las compañias que participan en las actividades de la "Iniciativa por la Opción en Software", sistemáticamente han objetado la aprobación de propuestas orientadas a impulsar la adopción de software de código abierto en la administración pública de diferentes gobiernos. Los casos de Venezuela, Colombia, Perú, así como los de algunos países de la Comunidad Europea han sido considerados como motivos de preocupación para ese organismo. En Venezuela, por ejemplo, como parte de las "Medidas de Reactivación Económica", y las "Políticas Básicas de Modernización del Eśtado", el 
gobierno del presidente Hugo Rafael Chávez Frías, ha propuesto incluir un decreto destinado a establecer el uso de software de fuente abierta en cada una de las dependencias gubernamentales.

Opuesta radicalmente a los intereses de "La Iniciativa por la Opción en Software", la "Fundación de Software Libre" promueve la necesidad de compartir y modificar los códigos fuente, afirmando que tal operación representa un legítimo derecho de los usuarios.

En México, el tema de la normatividad relativa al software a emplear por el Estado, representa una más de las asignaturas pendientes del gobierno del presidente Vicente Fox. Las lagunas jurídicas en la materia resultan muy delicadas. Por tal motivo Miguel de Icaza, principal promotor del uso de software libre en nuestro país señaló: "(...) no acuñar el e-México con software libre convierte a dicho plan nacional en un rehén tecnológico de las grandes multinacionales, ya que se crea una dependencia plena en cuestiones como seguridad informática, mejoras tecnológicas y los más osados apelan a la soberanía". De acuerdo con Miguel de Icaza, el gobierno de México por lo menos deberá pagar 3,500 millones de dólares a Microsoft por concepto de licencias y renovación de las computadoras que serán instaladas en las 1,200 bibliotecas públicas del país como parte de la iniciativa "Hacia un pais de lectores", la cual forma parte del Sistema Nacional e-México. Miguel de Icaza además afirmó que con los millones de dólares que podrían ahorrarse por el pago de las licencias que supone el uso del software propietario de Microsoft, el gobierno del presidente Vicente Fox bien podría entrenar y dar empleo a toda una generación de programadores, y crear una industria mexicana de software.

En el mes de junio de 2002, el Instituto Nacional para la Educación de los Adultos (INEA), anunció la intención de abrir mil centros de alfabetización, basados en internet, con una inversión estimada en mil millones de pesos. En Milenio.com, Hiroshi Takahashi reveló que los mexicanos que podrian estar interesados en vender tecnología y equipo al INEA, y participar en el proyecto de las Plazas Comunitarias e-México, deliberadamente fueron excluidos de la convocatoria del INEA:

Con fundamento en el artículo 23, fracción $\mathrm{I}$, inciso $\mathrm{b}$ ) del Reglamento de la Ley de Adquisiciones, Arrendamientos y Servicios del Sector Público y mediante el análisis de información elaborado por el instituto, se desprende que los productos nacionales no satisfacen adecuadamente las necesidades de los bienes requeridos por el INEA.

De acuerdo con Takahashi, en el anexo I de las bases de la referida licitación, la primera partida, destinada a cubrir 300 plazas comunitarias o centros de alfabetización, contempló la compra de 2,700 computadoras para estudiantes, y 300 para los profesores. Las tres mil computadoras que fueron solicitadas son marca Apple, modelo G3. Las computadoras, ade- 
más, deberían contar con el sistema operativo Mac OS, de Apple; programas de aplicación de Microsoft Office en español; Explorer y software preinstalado que permitiría la conversión de documentos de cualquier aplicación a formato PDF. La segunda partida, para 600 centros, contempló 5,400 computadoras Pentium 4 para los estudiantes, y 600 para los profesores. Cada computadora debería tener el programa Windows XP Pro, en español (de Microsoft), con licencia y disco compacto. Takahashi señaló que no fue estipulada la necesidad de ofrecer precios especiales para las computadoras de los estudiantes. Por lo anterior, es posible afirmar que en las computadoras que fueron solicitadas por el INEA para la creación y el equipamiento de los mil centros de alfabetización, definitivamente no podría ser utilizado software gratuito, a pesar de que ello representaría menores costos.

El caso de los mil centros de alfabetización del INEA, permite suponer que el gobierno del presidente Vicente Fox efectivamente ha correspondido a las generosas y "desinteresadas" donaciones de Bill Gates. Es posible inferir que el caso del INEA apenas representa la punta del icebeng.

\section{La VI Conferencia Internacional "El reto de México ante la Cumbre Mundial de la Sociedad de la Información". El escenario de la gran confrontación}

En la base misma de la discusión sobre la Sociedad de la Información, está el tema ineludible, indiscutible $-\mathrm{y}$ yo digo ineluctable - de nuestro país: la democratización de los derechos a la información y a la comunicación. Y para ello, no sólo se requiere garantizar el derecho, el acceso y el mayor acceso posible de todos los ciudadanos a las nuevas tecnologias para ir cerrando la brecha digital, sino cerrar otras brechas que amplian la exclusión de la mayoría de los ciudadanos para poder comunicarse, expresar lo que piensan y participar de ese intercambio que constituye todo proceso de comunicación [Javier Corral, 2003: 233].

Gracias a la iniciativa del senador Javier Corral Jurado (PAN), presidente de la Comisión de Comunicaciones y Transportes del Senado de la República, quien contó con el apoyo de la Asociación Mexicana de Derecho a la Información (AMEDI), la Fundación Konrad Adenauer Stiftung, la Universidad Autónoma Metropolitana (UAM), la Universidad Iberoamericana (UIA), y la Organización de las Naciones Unidas para la Educación, la Ciencia y la Cultura (UNESCO), del 28 al 30 de mayo de 2003, se realizó la VI Conferencia Internacional "El reto de México ante la Cumbre Mundial de la Sociedad de la Información", cuyo principal propósito era definir la posición que México debería asumir en la primera fase de la Cumbre Mundial de la Sociedad de la Información. 
En la VI Conferencia Internacional participaron más de 50 académicos, investigadores de la comunicación, legisladores, promotores de la libertad de expresión, consultores dedicados a la industria de las nuevas tecnologías, funcionarios públicos y periodistas, quienes analizaron los retos que supone el tránsito hacia la llamada Sociedad de la Información. El miércoles 28 de mayo de 2003 , en el acto de inauguración, el canciller Luis Ernesto Derbez afirmó que el gobierno federal tomaría en consideración las propuestas que fueran presentadas en la VI Conferencia Internacional, las cuales serían incorporadas a la iniciativa que México llevaría a la I Cumbre Mundial de la Sociedad de la Información, el mes de diciembre de 2003, en Ginebra, Suiza.

La VI Conferencia Internacional, desafortunadamente, fue marco de enfrentamientos y exclusiones. Heidi Storsberg Montes, por ejemplo, entonces diputada federal del PAN, y presidenta de la Comisión Bicameral del Canal del Congreso, fue marginada de las actividades de la VI Conferencia Internacional, en razón de las sensibles diferencias que prevalecian entre la diputada Heidi Storsberg y el senador Javier Corral. La diputada Heidi Storsberg había impulsado la destitución de Virgilio Dante Caballero Pedraza, director general del Canal del Congreso. Felipe Calderón, el entonces líder de la bancada panista en la Cámara de Diputados, en no pocas ocasiones había expresado inconformidad por el desempeño de Virgilio Caballero al frente del Canal del Congreso.

El 16 de diciembre de 2002, Beatriz Paredes, diputada federal (PRI), y presidenta de la Cámara de Diputados, sorpresivamente dio a conocer la destitución del director del Canal del Congreso, Virgilio Caballero, "por desacato a las ordenes de sus superiores". Tal destitución fue calificada en el Senado de la República como un acto ilegal. Algunos senadores argumentaron que el canal de televisión del Congreso debería ser reconocido como entidad bicamaral, y conforme a lo dispuesto en el artículo 132 de la Ley Orgánica del Congreso de la Unión, la Comisión Bicameral es la única autoridad facultada para manejar el canal, y autorizada para designar o destituir a su director. El 20 de febrero de 2003, Virgilio Caballero finalmente presentó su renuncia ante la Comisión Bicamaral del Poder Legislativo, la cual fue aceptada por tres de sus seis integrantes. El semanario Proceso con anterioridad había consignado presuntas irregularidades cometidas por Virgilio Caballero en el manejo del Canal del Congreso.

Apenas unas horas antes del acto inaugural de la VI Conferencia Internacional, funcionarios de la Secretaría de Comunicaciones y Transportes (scT), y de la Comisión Federal de Telecomunicaciones (Cofetel), notificaron a los organizadores su decisión de no participar. El senador Javier Corral calificó el proceder de los funcionarios de las referidas instituciones, como resultado de un "berrinche de Pedro Cerisola", titular de la SCT. El senador Javier Corral cuestionó hasta la autonomía de Cofetel, al señalar que su titular había aceptado ordenes del arquitecto 
Pedro Cerisola, secretario de Comunicaciones y Transportes.

De acuerdo con Alejandro Gutiérrez, pertiodista del semanario Proceso, el senador Javier Corral, quien fue candidato - perdedor- al gobierno de Chihuahua por la coalición "Todos somos Chihuahua", la cual integraron el Partido Acción Nacional (PAN), el Partido de la Revolución Democrática (PRD), y Convergencia Democrática (CD): "es hijo político de Francisco Barrio, ex gobernador y hoy coordinador parlamentario del PAN en la Cámara de Diputados" (Gutiérrez, 2004: 29).

En la VI Conferencia Internacional, "El reto de México ante la Cumbre Mundial de la Sociedad de la Información" fueron expresados enérgicos cuestionamientos al Sistema Nacional e-México, el cual, se encuentra a cargo de la SCT, cuyo titular precisamente es Pedro Cerisola. Scott Robinson, por ejemplo, profesor e investigador del Departamento de Antropología de la Universidad Autónoma Metropolitana, Unidad Iztapalapa, presento la ponencia "El reto inmediato: reconfigurar el programa e-México". Robinson aseveró que el proyecto e-México, tal y como ha sido concebido y desarrollado, no tiene remedio:

[...] estas alturas, el único camino para que tengamos una política informática nacional sustentada por el Estado y la sociedad, es la construcción de un nuevo proyecto. Para sustituir a e-México, cuyo fracaso se advierte desde la ausencia de metas claras y se ratifica en la ubicación de ese proyecto en la Secretaría de Comunicaciones y Transportes y no en la SEP o en el Conacyt, se propuso crear un comité de especialistas que diseñe, en pocos meses, el programa y una ruta para el desarrollo nacional de internet en México. Ese comité podría funcionar a semejanza del que hace tres años elaboró en Brasil el Programa Sociedad de la Información [Trejo, 2003: 271].

A diferencia de los funcionarios de SCT y Cofetel, quienes efectivamente fueron invitados a presentar ponencias en la VI Conferencia Internacional, y que de acuerdo con el senador Javier Corral, en el último momento decidieron no participar, por obedecer "instrucciones superiores", determinadas personalidades de la academia de las ciencias de la comunicación en México, quienes han estudiado el tema de la Sociedad de la Información, como la maestra Carmen Gómez Mont, la doctora Delia Crovi, el doctor Ernesto Villanueva, por ejemplo, no fueron considerados por los organizadores de la VI Conferencia Internacional. Además, fueron excluidas determinadas instituciones y organismos, a pesar de sus relevantes aportaciones al desarrollo de internet en México. Entre los sensibles ausentes cabe mencionar al Tecnológico de Monterrey, AMPCI, y la CUDI, por citar algunos.

El 28 de febrero de 1989, el Tecnológico de Monterrey, Campus Monterrey, se convirtió en la primera institución en Iberoamérica que consiguió establecer un enlace dedicado a la red de la National Science 
Foundation (NSP), luego internet, a través de la Universidad de Texas en San Antonio (UTSA), Estados Unidos. Por esa sencilla razón, el Tecnológico de Monterrey dispone del primer "name server" para el dominio ".mx". En febrero de 1989, el Stanford Research Institute Network Information Center (SRI-NIC), con la aprobación de Jon Postel, entonces responsable de asignar la administración de dominios nacionales, delegó en el Tecnológico de Monterrey, Campus Monterrey, la administración del "country code Top Level Domain" o ccTLD .MX. De acuerdo con información publicada en el sitio www de NIC-México:

El Network Information Center-México, (NIC-México) es la organización encargada de la administración del nombre del dominio territorial (ccTD, country code Top Level Domain) .Mx, el código de dos letras asignado a cada país según el iso 3166. Entre sus funciones están el proveer los servicios de información y registro para MX así como la asignación de direcciones de IP y el mantenimiento de datos respectivos a cada recurso. Éste nace el primero de febrero de 1989, cuando el ITESM, Campus Monterrey establece conexión directa a internet.

El ingeniero Oscar Robles, catedrático del Tecnológico de Monterrey, Campus Monterrey, se desempena como director general del Centro de Servicios de Información y Registro en Internet (Network Information Center de México o NIC-México). A pesar de la importante tarea que desempeña en la administración de los recursos de Internet en México, los organizadores de la I Conferencia Internacional, no repararon en invitärlo a presentar sus opiniones, indudablemente valiosas, en la posición que presentaría México en la I Cumbre Mundial de la Sociedad de la Información.

El Tecnológico de Monterrey es una de las instituciones que más han impulsado el desarrollo de Internet 2 en México, y el ingeniero Juan José Zamanillo, investigador del Sistema Tecnológico de Monterrey, es presidente de la Corporación Universitaria para el Desarrollo de Internet, A.C. (CUDI). Ese organismo se encuentra a cargo del desarrollo del Proyecto internet 2 en México. Sobre la importancia de internet 2, el doctor Roberto Gómez, experto en seguridad computacional y destacado investigador del Tecnológico de Monterrey, Campus Estado de México, afirma:

Internet-2 surge en los Estados Unidos durante la administración de Bill Clinton con el movimiento denominado "Iniciativa para un Intemet de Nueva Generación" (Next Generation Internet Initiative), con el propósito de fomentar la conectividad de la más alta capacidad entre centros de investigación y universidades. La principal diferencia entre internet e Internet-2 está en la velocidad en la que se transmite la información y en el hecho de que las aplicaciones desarrolladas en esta red utilizarán un completo conjunto de herramientas que no existen en la actualidad. Desde el punto de vista de velocidad, encontramos velocidades de 2 Gbps en la 
parte medular de la red y las conexiones de las universidades a esta sección varian entre $45 \mathrm{Mbps}$ y $622 \mathrm{Mbps}$. Entre las herramientas con las que cuentan los desartolladores podemos mencionar lo que se conoce como calidad de servicio, una mayor preocupación por la seguridad y un soporte técnico de primera calidad. Existen varios proyectos que se están llevando a cabo en Internet-2, destacando telemedicina, bibliotecas digitales, laboratorios virtuales, manipulación a distancia y visualización de modelos $3 \mathrm{D}$; aplicaciones que aprovechan las ventajas de la velocidad de la nueva red y de las herramientas disponibles. En México, el organismo encargado de manejar el proyecto de Internet-2 es la Corporación Universitaria para el Desarrollo de Internet, A.C. (CUDI). La CuDI es una asociación civil privada no lucrativa de instiuciones acadénicas, la cual se consolida el 8 de abril de 1999 [...]. Actualmente, la CuDt está conformada por 50 miembros de diferentes universidades y centros de investigación. Podemos mencionar a la UNAM, el Tec de Monterney, la UAM, el IPN, la ULSA, entre orras universidades. Del lado de los centros de investigación podemos mencionar al Cinvestav, el LANIA, el IMP, CICESE y otros. A nivel internacional se cuenta con varios proyectos similares. En los Estados Unidos se cuenta con Internet-2, en Canadá el proyecto $\mathrm{CA}^{*}$ net3, en Europa los proyectos TEN155 y GEANT, y en Asia el proyecto APAN. Adicionalmente, todas estas redes están conectadas entre sí, formando una gran red avanzada de alta velocidad de alcance mundial. En Latinoamérica, las redes académicas de México cuDI, Brasil, Argentina RETINA y Chile REUNA ya se han integrado a Intemet-2.

A pesar de la importancia de Internet-2 en la Sociedad de la Información, el tema apenas fue mencionado en la VI Conferencia Internacional.

La Asociación Mexicana de Internet (AMPCI), organismo en el cual participan las principales empresas dedicadas al desarrollo comercial de internet en México, tampoco fue invitada a participar en la VI Conferencia Internacional. Estas son algunas de las instituciones que se encuentran afiliadas a AMIPCl, activamente: Avantel, Banamex, Bancomer, Bancomext, Bital, Certifica.com, CNI en línea, Diveo, EsMas.com, Liverpool, NIC-México, Proceso, Prodigy Internet, Real Networks, Respuesta Creativa, Santander Serfin, ScotiaBank Inverlat, T1MSN, Tecnológico de Monterrey, Terra Network, Teve de Mente, Yahool, El Universal.

A través de su vicepresidencia jurídica, la cual se encuentra a cargo del destacado abogado Luis Vera, AMIPCI ha realizado relevantes contribuciones a las siguientes iniciativas de ley:

- Ley del Comercio Electrónico -el decreto del 29 de mayo de 2000-, la cual implicó reformas y adiciones al Código Civil Federal, al Código de Comercio, Código Federal de Procedimientos Civiles, Ley Federal de Protección al Consumidor.

- La nueva legislación de firmas para el comercio electrónico a través de internet -la cual supone reformas de los artículos 96 al 114 del Código de Comercio (Federal)-. 
- Reformas y adiciones a la Ley Federal del Derecho de Autor.

- Ley Federal de Protección de Datos Personales.

- Reformas a la Ley Federal de Protección al Consumidor.

- Reformas al Código Fiscal de la Federación para el reconocimiento y validez de la Factura Electrónica tanto en operaciones comerciales entre empresas (Business to Business o B2B) o de empresas al consumidor (Business to Consumers o B2C), así como su incorporación en los comprobantes fiscales electrónicos que aceptará el sat.

AMPCI además ha realizado algunas de las más relevantes investigaciones sobre hábitos culturales y prácticas de consumo de los cibernautas en México. No obstante, los organizadores de la VI Conferencia Internacional, olvidaron extender una invitación a AMIPCI para participar en las actividades de la VI Conferencia Internacional.

$\mathrm{La}$ VI Conferencia Internacional "El reto de México ante la Cumbre Mundial de la Sociedad de la Información", no sólo se distinguió por desafortunadas exclusiones, las cuales naturalmente nos obligan a dudar de la sinceridad de los pronunciamientos a favor de "una sociedad democrática e incluyente ${ }^{n}$. Peor aún, la VI Conferencia Internacional evidenció cuán profundas ya resultaban algunas diferencias, fundamentalmente políticas, entre algunos grupos de poder, tanto de panistas como de funcionarios del gobierno del presidente Vicente Fox, dispuestos a imponer sus particulares intereses, aun a costa de conducir al fracaso la iniciativa estelar del gobierno foxista en materia de e-Gobierno.

\section{La respuesta de Pedro Cerisola}

Para atajar los duros cuestionamientos que recibio el Sistema Nacional eMéxico en la VI Conferencia Internacional "El reto de México ante la Cumbre Mundial de la Sociedad de la Información", en julio de 2003, los administradores del sitio www del Sistema Nacional e-México, por fin publicaron en internet su visión de la Sociedad de la Información:

Es una iniciativa en la que se vienen sumando la mayor parte de los países, para apoyar el desarrollo humano mediante el uso de las tecnologias de información y comunicaciones, generando oportunidades para el desarrollo de servicios en el aprendizaje, la salud, la economía, el gobierno y orros servicios afines. Para que más población tenga acceso a servicios, en un entorno colaborativo de alcance local, regional, nacional e internacional, se contempla el impulso a las tecnologías diversas de información y comunicaciones, que permitan no sólo la innovación y modernización de infraestructura, sino también la homologación y/o armonización de las normas en cada región, para apoyar un entorno internacional de intercambio de información y conocimiento, apoyado en una conectividad de alcance 
global. En el ámbito educativo y para lograr el desarrollo de habilidades que apoyen la superación del individuo, como agente catalizador de la sociedad, se requiere el aprovechamiento de los servicios de aprendizaje, al incorporar los elementos de cultura local y la identidad nacional, así como utilizar esquemas de educación que contemplen desde los conocimientos básicos de un sistema escolar, integrando elementos para aprender a aprender, hasta el desarrollo de una currícula acorde a las expectativas de cada pais; con los esquemas de capacitación y aprendizaje continuo a lo largo de la vida. Existen estudios que valoran las bondades del uso de estas tecnologías para cubrir un mayor número de personas, ofreciendo una mejor atencion. Generando con esto, una mejora en las condiciones de vida de la familia y su comunidad. En el ámbito de los servicios de salud y para atender a un mayor número de pacientes, con más calidad en la atención y proporcionando mejor seguimiento en cada caso. Experiencias internacionales han demostrado que con el uso adecuado de estas tecnologías aumenta el volumen de atención médica y la calidad en los servicios de salud, aprovechando las tecnologías para ofrecer, entre otros, servicios a distancia empleando la Telesalud, integrando programas para la actualización y capacitación del personal de los servicios en este sector, así como los sistemas para mejorar la administración y gestión de las unidades médicas, permitiendo sinergias entre las instituciones y dependencias, al generar esquemas de colaboración entre el sector público y el sector privado, lo que se verá reflejado en la mejora de los índices e indicadores de salud. En el ámbito de la economía y para la mejora de los procesos de las empresas pequeñas y medianas, por ser éstas las mayores empleadoras y la principal fuente de riqueza social, al incorporar los sistemas de administración de uso de recursos, mediante las tecnologías que permiten la optimizacion de las cadenas de valor para lograr una mejor eficiencia. Establecer una política nacional para apoyar el desarrollo de empresas del conocimiento, de programación y servicios afines, que permitan mayores ingresos y empleos de mayor remuneración. En un país no basta con utilizar las nuevas tecnologías, es indispensable participar en la innovación tecnológica mediante el aprovechamiento de las redes de conectividad, siendo esto un imperativo. Los gobiernos tienen que atender a los ciudadanos del siglo $\mathrm{xxI}$, con servicios del siglo $\mathrm{xx}$. Los servicios gubernamentales requieren de incorporar las mejores prácticas del uso de las tecnologías de información y comunicaciones, para aprovechar de manera directa sus beneficios y minimizar los costos crecientes que se presentan. Como una mecánica para agilizar la modernización de los servicios gubernamentales en este ámbito de transformación de los gobiernos locales, regionales y nacionales, se requiere de una participación y un intercambio de las diversas experiencias mundiales, para proponer y aprender con los demás países, las acciones estratégicas y los compromisos que permitan mejorar las condiciones de vida de la población, promoviendo en todos los ámbitos, ta gestión inteligente de las infraestructuras y la coordinación de las dependencias, instituciones y organismos afines; realizando el control, inspección y aplicación de las políticas de desarrollo, lo que permite generar una mayor agilidad, transparencia y seguridad de los trámites y servicios. El despliegue 
de la Sociedad de la Información, contempla también la creación de servicios diversos de atención ciudadana, como los servicios especializados en las bolsas de trabajo, servicios, turísticos, apoyo a las actividades de investigación y desarrollo, promoción y fomento al cuidado del medio ambiente, atención a los grupos migrantes y minorias, asi como la creación de modelos de teletrabajo y otros. La sociedad de la información es, en suma, fomentar el acceso de los ciudadanos a la informacion, ampliando la cobertura y alternativas de comunicación, así como mejorar la calidad de los servicios al ciudadano, ofreciendo nuevas oportunidades de desarrollo humano, mediante la innovación, aprovechando las tecnologías de información y comunicaciones.

Durante los primeros días de junio de 2003, en el Palacio Postal de la ciudad de México, fue realizada la primera conferencia del presidente Vicente Fox con los 31 estados de la República, y el Distrito Federal, a través del Sistema Nacional e-México. En el acto, el presidente Vicente Fox refrendo la confianza depositada en el secretario Pedro Cerisola, a quien ratifico como líder indiscutible del Sistema Nacional e-México. El presidente Vicente Fox además aprovechó esa oportunidad para destacar las acciones que ha emprendido su administración en materia de gobierno digital: "México fue seleccionado como el gobierno que más avanzó en materia de gobierno digital entre todas las naciones del mundo".

Sin precisar quiénes habían seleccionado a México como el gobierno que más avanzó en 2003 en materia de gobierno digital, el presidente Fox afirmó que ya es posible realizar a través de internet 420 trámites públicos del gobierno federal; anunció un interesante programa de preparatoria abierta a travếs de internet, y destac 6 que a la fecha se han establecido 3,200 centros comunitarios digitales en escuelas, bibliotecas, centros de salud, centros de desarrollo social, plazas comunitarias, presidencias municipales y oficinas de correos y telégrafos. El presidente Vicente Fox también anunció que en 2004, el número de centros comunitarios digitales crecería a 10,000 .

La optimista visión del presidente Fox, de ninguna manera corresponde con los resultados de la medición realizada por el "World Economic Forum", la cual destacaba que en materia de desarrollo tecnológico, México había pasado de la cuadragésima cuarta posición mundial -en el año 2002-, a la cuadragésima séptima posición, en el año 2003. Las evaluaciones obtenidas por Brasil, Chile, y Argentina fueron superiores a la alcanzada por México (Piedras, 2003: 20).

Vale la pena destacar que del 21 al 23 de agosto de 2003, la Universidad Nacional Autónoma de México (UNAM), realizó el "Primer Foro Sociedad de la Información ¿Qué haremos?" El organizador, Alejandro Pisanty, es responsable de Sistemas Computacionales de la UNAM. El doctor Julio César Margáin, entonces director del Sistema Nacional e-México, dictó una de las conferencias magistrales y también participó en la relatoría de las 
conclusiones. En esa ocasión, el senador Corral, quien había confirmado su participación a los organizadores, en el último momento canceló. Un alto funcionario de la Secretaría de Comunicaciones y Transportes, no sin cierta ironía, expreso: "Se trató del berrinche del senador Corral".

\section{El previsible desencanto que arrojó la Cumbre Mundial de la Sociedad de la Información}

Naciones Unidas es una de las organizaciones que más esperanzas ha fincado en la edificación de la "Sociedad de la Información". Por tal motivo, realizó del 10 al 12 de diciembre de 2003, en Ginebra, Suiza, la primera fase de la Cumbre Mundial sobre la Sociedad de la Información. Túnez será sede de la segunda fase de la Cumbre Mundial, del 16 al 18 de noviembre de 2005. De acuerdo con Naciones Unidas, los antecedentes de la Cumbre Mundial sobre la Sociedad de la Información son los siguientes:

La Resolución 73 de la Conferencia de Plenipotenciarios de la urr (Minneápolis, 1998) resolvio encargar al Secretario General de la unt inscribir en el orden del día del Comité Administrativo de Coordinación ( $\mathrm{CAC}$ ), ahora Junta de Jefes Ejecutivos (bE) del sistema de las Naciones Unidas, que pasó a denominarse Junta de Jefes Ejecutivos del Sistema de las Naciones Unidas para la Coordinación (CEB), la cuestión de la celebración de una Cumbre Mundial sobre la Sociedad de la Información, e informar al ente directivo de la UT, el Consejo, sobre los resultados de dicha consulta. El Secretario General indico en su Informe a la sesión del Consejo de 1999, que el cac tuvo una reacción positiva y que la mayoráa de las otras organizaciones y organismos expresaton interés en asociarse con la preparación y celebración de la Cumbre. Se decidio que la Cumbre sería celebrada bajo el auspicio de la Secretaría General de la ONU, y que la uTT asumiría la intervención principal en los preparativos. En 2001, el Consejo de la urT decidió celebrar una Cumbre en dos etapas, la primera en Ginebra (Suiza), del 10 al 12 de diciembre de 2003, y la segunda en Túnez (Túnez), del 16 al 18 de noviembre de 2005. En la Resolución 56/183 de la Asamblea General de las Naciones Unidas se aprobó el marco de la Cumbre adoptado por el Consejo de la unt así como la función principal de la Unión en la Cumbre y su proceso preparatorio, en cooperación con otras organizaciones y asociados interesados. La Resolución 56/183 de la Asamblea General de las Naciones Unidas recomienda que se encarguen los preparativos de la Cumbre a un Comité Preparatorio Intergubernamental de composición abierta, que establecerá el programa de la Cumbre, decidirá las modalidades de participación de otros interesados en la Cumbre y concluirá la redacción del proyecto de declaración y el proyecto de plan de acción. Se invita a la urr a asumir la función administrativa principal de la Secretaría Ejecutiva de la Cumbre $y$, asimismo, se invita a los gobiernos a participar activamente en el proceso 
preparatorio de la Cumbre y a enviar a ella representantes del más alto nivel. En la Resolución 56/183, la Asamblea General alienta, asimismo, a todos los organismos competentes de las Naciones Unidas y, en particular, al Grupo Especial de las Naciones Unidas sobre las TIC, a aportar contribuciones. Alienta además a otras organizaciones intergubernamentales y, en particular, a las instituciones internacionales y regionales, las organizaciones no gubernamentales, la sociedad civil y el sector privado, a participar activamente en el proceso preparatorio intergubernamental de la Cumbre y en la propia Cumbre.

A finales de 2000, los estados miembros de Naciones Unidas definieron los "Objetivos de desarrollo de la onu para el milenio" —que también son conocidos como "Metas de desarrollo de la Declaración del Milenio-. Los ocho objetivos que los 191 estados miembros de Naciones Unidas se comprometieron alcanzar en el año 2015, son:

1. Erradicar la pobreza extrema y el hambre.

2. Lograr la enseñanza primaria universal.

3. Promover la igualdad entre los géneros y la autonomía de la mujer.

4. Reducir la mortalidad infantil.

5. Mejorar la salud materna.

6. Combatir el viH/SIDA, el paludismo y otras enfermedades.

7. Garantizar la sostenibilidad del medio ambiente.

8. Fomentar una asociación mundial para el desarrollo.

Cada una de las "Metas de desarrollo de la Declaración del Milenio" representa el fundamento último de determinados objetivos particulares. En el objetivo número 18 de la octava meta: "Fomentar una asociación mundial para el desarrollo", se reconoce la importancia de las TIC en las sociedades del nuevo milenio: "En colaboración con el sector privado, velar por que se puedan aprovechar los beneficios de las nuevas tecnologías, en particular, los de las tecnologías de la información y de las comunicaciones". Como se puede advertir en el cuadro 1, la cual fue elaborada, a partir de la información que es posible consultar en el sitio www de la Unión Internacional de Telecomunicaciones (UIT), el inteligente empleo de las TIC podría contribuir al logro de los "Objetivos de desartollo de la Declaración del Milenio:

En uno de los documentos más relevantes que derivaron de la primera fase de la Cumbre Mundial sobre la Sociedad de la Información -la Declaración de Principios, Construir la sociedad de la información: un 
CUADRO 1 Posible incidencia del inteligente empleo de las $T \mathrm{C}$ para alcanzar lo dispuesto en los "Objetivos de Desarrollo de la ONU para el Milenio"

\begin{tabular}{lll}
\hline Objetivos & Indicador & Incidencia \\
\hline
\end{tabular}

$\begin{array}{ll}\text { Erradicar } & \text { Aumento de } \\ \text { la pobreza } & \text { los ingresos } \\ \text { extrema } & \text { provenientes } \\ \text { y el hambre } & \text { de las TIC }\end{array}$

Lograr la enseñanza primaria universal

Promover la igualdad entre los géneros y la autonomía de la mujer
Reducir la mortalidad infantil
Capacitación de maestros de escuela primaria mediante las ThC
Porcentaje de mujeres matriculadas en programas de educación que utilizan las TIC en relación con el número total de mujeres matriculadas a nivel superior
Según un estudio realizado en 1999 sobre los propietarios de teléfonos de previo pago en las aldeas de Bangladesh, $24 \%$ de los ingresos totales de esos hogares provienen de la prestación del servicio telefónico.

En Nepal, 4,430 personas recibieron en 2001 una capacitación a distancia como maestros de escuelà primaria a través de sistemas de radiocomunicaciones. Tomando como base las cifras actuales de un maestro para 40 estudiantes, podrian inscribirse 176,616 nuevos alumnos en ese tipo de escuela cuando los maestros completen su formación. El aumento neto del número de inscripciones en escuelas primarias sería del $5,7 \%$.

En Australia, el proyecto Open Learning Australia (OLA) ofrece un nivel superior de educación que combina la enseñanza a distancia y la enseñanza en línea. En 2002, 6,129 estudiantes, de los cuales el $56.9 \%$ eran mujeres, participaron en este programa. La proporción de alumnas es superior a la que existe en la enseñanza secundaria en general ( $54.9 \%$ ). Como resultado del programa OLA, la tasa de matriculación de alumnas a nivel superior es $0.8 \%$ más elevada.

Baby CareLink es un programa de telemedicina para padres de niños pequeños en los Estados Unidos. Según una evaluación realizada en 1997-1999 sobre 56 pacientes, los padres que utilizan Baby CareLink proporcionan a sus hijos cuidados de una calidad $10 \%$ superior en comparación con los padres que no lo hacen. 
CUADRO 1 (concluye)

\begin{tabular}{ll}
\hline \multicolumn{1}{c}{ Objetivos } & \multicolumn{1}{c}{ Indicador } \\
\hline $\begin{array}{l}\text { Mejorar la } \\
\text { salud materna }\end{array}$ & $\begin{array}{l}\text { Porcentaje de } \\
\text { trabajadores que } \\
\text { utilizan las TIC en el } \\
\text { ámbito de la salud } \\
\text { maternal }\end{array}$ \\
Combatir el & $\begin{array}{l}\text { Porcentaje de } \\
\text { población adulta que } \\
\text { vit/siDA, el } \\
\text { paludismo y } \\
\text { otras }\end{array}$ \\
enfermedadesta un modo de \\
vida más sano tras \\
haber recibido, por \\
medio de las TIC, \\
información sobre la \\
salud
\end{tabular}

Garantizar la sostenibilidad del medio ambiente
Porcentaje de teletrabajadores en relación con el número total de trabajadores
Incidencia

Según la evaluación de un proyecto de salud maternal basado en las tecnologías de las radiocomunicaciones realizada en julio de 1999 en el distrito de Tororo (Uganda), se constató que la tasa de mortalidad maternal se redujo a la mitad.

Tras la evaluación realizada en septiembre de 1998 de un programa radiofónico educativo sobre la prevención del viH en Santa Lucía, la importación de preservativos aumentó $143 \%$ tras la difusión del programa.

Hay 38700 teletrabajadores en Irlanda, es decir el $2,3 \%$ del número total de trabajadores. Como consecuencia, las emisiones de dióxido de carbono provenientes del tráfico automotriz han disminuido el $2 \%$. Si los irlandeses cuyas actividades profesionales muestran una tendencia al teletrabajo (28\% del número total de trabajadores) trabajarán a domicilio, las emisiones de dióxido de carbono disminuirían el $30 \%$.

FueNTE: Unión Internacional de Telecomunicaciones, Informe sobre el Desarrollo Mundial de las Telecomunicaciones (urr, 2003). Medir el acceso a la sociedad de la información. Evaluar la incidencia de las tic en los Objetivos de Desartollo establecidos a escala mundial. 23 indicadores e para reducir las brechas digital y estadistica.

desafio mundial para el nuevo milenio-, 18 enunciados -del total de 67-, corresponden al propósito de establecer la visión de Naciones Unidas sobre la "sociedad de la información":

1. Nosotros, representantes de los pueblos del mundo, reunidos en Ginebra del 10 al 12 de diciembre de 2003 con motivo de la primera fase de la Cumbre Mundial sobre la Sociedad de la Información, declaramos nuestro deseo y compromiso comunes de construir una sociedad de la información centrada en la persona, incluyente y orientada al desarrollo, en la que todos puedan crear, consultar, utilizar y compartir la información y el conocimiento, para que las personas, las comunidades y los pueblos puedan desarrollar su pleno potencial en la promo- 
ción de su desarrollo sostenible y mejorar su calidad de vida, de acuerdo con los objetivos y principios de la Carta de las Naciones Unidas y espetando y defendiendo plenamente la Declaración Universal de Derechos Hunanos.

2. Nuestro desafio es encauzar el potencial de la tecnología de la información y la comunicación para promover las metas de desarrollo de la Declaración del Milenio, a saber, erradicar la extrema pobreza y el hambre, lograr una educación primaria universal, promover la igualdad de género y la habiitación de las mujeres, reducir la mortalidad infantil, mejorar la salud materna, combatir el vH/sIDA, el paludismo y otras enfermedades, garantizar la sustentabilidad ambiental y forjar alianzas mundiales en favor del desarrollo para lograr un mundo más pacífico, justo y próspero. Reiteramos asimismo, nuestro compromiso fara con el logro del desarrollo sostenible y las metas de desarrollo convenidas, que se señalan en la Declaración de Johannesburgo y en el Plan de Aplicación del Consenso de Monterrey, y otros resultados de las Cumbres pertinentes de las Naciones Unidas.

Si bien en los "Objetivos de desarrollo de la onU para el milenio", es reconocida la relevante contribución que podría representar el inteligente y generoso empleo de las avanzadas tecnologías de infcrmación y comunicaciones, para la edificación de un mundo más justo e igualitario, debemos tener muy presente que en la actualidad $90 \%$ de la población mundial, prácticamente se encuentra excluida de los beneficios que supone la llamada "Sociedad de la Información".

Por otra parte, los primeros atentados hiperterroristas de la historia, registrados el 11 de septiembre de 2001, modificaron las prioridades de la administración del presidente George W. Bush, quien deiidió apalancar el desarrollo de la economía estadounidense en dos negocios que de ninguna manera resultan ajenos a los intereses económicos de su acaudalada familia: el petróleo y la guerra. El tránsito a la "Sociedad de la Información" entró así en un pronunciado suspenso. Por lo pronto, la única posibilidad inmediata que permitiría reubicar la importancia de la Sociedad de la Información en el panorama político internacional, dependerá del resultado que arrojen los próximos comicios presidenciales en los Estados Unidos (noviembre, 2004). La continuidad de Bush, no sólo aparta al mundo de la grata utopía que podría representar la Sociedad de la Información, su reelección representa una grave amenaza a la paz mundial.

A pesar del desencantamiento de la primera reunión de la Cumbre Mundial sobre la Sociedad de la Información, en diciembre de 2003, más de 11,000 personas acudieron al Palexpo de Ginebra, para asistir a un evento de gran trascendencia internacional, al cual supvestamente asistirían los jefes de Estado. El acto finalmente fue transformado en una gran feria mundial, la cual contó con más de 250 pabellones para la exhibición de productos de empresas, determinadas iniciativas de los países asistentes, y hasta de los organismos no gubernamentales que disponían de los recursos necesarios para rentar los espacios. 
Representantes de 176 gobiernos, y más de 3,000 miembros de 481 organizaciones no gubernamentales, presentaron propuestas sobre el posible rumbo que podría seguir la "Sociedad de la Información", en una especie de diálogo de sordos. En alguna ocasión, se realizaron, inclusive, más de 40 eventos de manera simultánea. En síntesis, los resultados que arrojo la primera reunión de la Cumbre Mundial sobre la Sociedad de la Información, fueron decepcionantes. Fue imposible alcanzar definiciones medulares en dos temas fundamentales: el gobierno de internet, y la creación de un Fondo de Solidaridad Digital para África.

La Internet Corporation for Assigned Names and Numbers (ICANN), organismo que controla aspectos fundamentales en la administración de internet, que tiene su sede en California, y que reporta al Departamento de Comercio de los Estados Unidos, seguirá administrando la asignación de números IP y nombres de dominio en el mundo, hasta el 2006, a pesar de las objeciones que presentaron representantes de los gobiernos de Brasil, Sudáfrica e India, quienes fundamentaron la necesidad de ceder el control de internet a un organismo dependiente de Naciones Unidas.

Por otra parte, la creación de un Fondo de Solidaridad Digital para África, iniciativa que presentó Abdoulaye Wade, presidente de Senegal, fue objetada por representantes de los Estados Unidos, la Union Europea, y Canadá. Como reconoce el destacado investigador y periodista Raúl Trejo Delarbre: "Habrá que esperar al encuentro de Túnez para saber en qué medida la algarabía de expresiones e intereses que circulan a propósito de la información puede traducirse en decisiones capaces de beneficiar a las grandes mayorias del planeta". (Trejo, 2004: 158).

La representación oficial del gobierno de México corrió a cargo del secretario de Comunicaciones, Pedro Cerisola, y el subsecretario Jorge Alvarez Hoth. Cerisola y Álvarez Hoth realizaron esfuerzos extraordinarios para justificar la ausencia de una efectiva estrategia de gobierno del presidente Vicente Fox en materia de gobierno digital. A comienzos de 2004, el doctor Julio César Margáin renunció a la dirección del Sistema Nacional e-México. El nuevo director es Javier Pérez Mazatán, quien resultó el ganador de la "rifa del tigre".

\section{Volver a empezar}

De acuerdo con Neil Postman, quien con base en el pensamiento de Marshall McLuhan fundó una importante escuela dedicada al estudio de la comunicación, conocida como Media Ecology, la introducción de toda nueva tecnología, así como el poner en marcha un ambicioso programa de innovación tecnológica, nos obliga a reflexionar en seis preguntas fundamentales: 
1. ¿Qué problemas es posible solucionar con la introducción de una nueva tecnología o con el desarrollo del programa de innovación tecnologica?

2. ¿De quién es el problema?

3. ¿Qué actores y qué instituciones podrían resultar afectados por la introducción de la nueva tecnología o con el desarrollo del programa de innovación tecnológica?

4. ¿Qué nuevos problemas podría producir la introducción de la nueva tecnología o el desarrollo del programa de innovación tecnológica?

5. ¿De qué manera la introducción de la nueva tecnología o el desarrollo del programa de innovación tecnológica, transformaría la ecología del aprendizaje de la sociedad?

6. ¿Qué actores y qué instituciones podrían adquirî́ poder económico o político, beneficiándose por la introducción de la nueva tecnología o con el desarrollo del programa de innovación tecnologica?

Dar respuesta a esas preguntas, permitirá dar el primer paso seguro para rectificar el rumbo del Sistema Nacional e-México. Es indispensable hacerlo. De lo contrario, habriamos perdido una gran oportunidad histórica. También, resulta indispensable reubicar al ciudadano como fundamento de todos los esfuerzos que realice el Estado en materia de e-Gobierno, tal como refería Al Gore.

El importante reconocimiento internacional con el cual fue distinguido el portal ciudadano que desarrolló el equipo de Abraham Sotelo Nava, en la Secretaría de la Función Pública [http://www.gob.mx], ganador del Premio Stockholn Challange 2003-2004, considerado como el "Nobel de Internet", en la categoría de e-Gobierno, confirma que el ciudadano debe ser la razón y fundamento de cada uno de los esfuerzos que realice el Estado en materia de e-Gobierno.

A pesar de que en el propio equipo del presidente Vicente Fox, algunos funcionarios públicos y destacados panistas "históricos" desafortunadamente han observado un desempeño que permitiría ubicarlos en el grupo de obstaculizadores que "hasta practican una especie de sabotaje", el trabajo que realiza una minoría efectivamente comprometida en la tarea de transformar la administración pública, permite mantener el país relativamente a flote. Ello permite renovar esperanzas. El porvenir del Sistema Nacional e-México dependerá de ubicar al ciudadano como su razón de ser. Si fuera por concepto y resultados, el Sistema Nacional e-México definitivamente debería ser reubicado en la Secretaría de la Función Pública. 


\section{Bibliografia}

Acosta, N. (2002), "Ponderan software gratuito", en El Unitersal, viernes 29 de noviembre, $\mathrm{B} 7$.

Arredondo, J. (2003), "México, líder en gobiemo digital: Fox", en El Universal, Computación, lunes 9 de junio, p. 1.

Bartolomé, F. (2001), "Templo Mayor", en Reforma, 1 de agosto, p. 22.

Bell, D. (1991), El advenimiento de la sociedad postindustrial, Alianza, Madrid.

Cantú, G. (2001), Asalto a palacio. Las entrañas de una guerra, Grijalbo, México.

Cebriån, J. (1998), La red, Taurus, España.

Comisión Europea (1993), Libro Blanco. Crecimiento, competitividad, empleo. Retos y pistas para entrar en el siglo XXI, Comunidad Europea, Luxemburgo.

Contreras, F. y C. San Nicolás (2001), Comunicactón, diseño grafico, creatitidad y comunicación, Blur Ediciones, Madrid.

Corral, J. (2003), "La necesidad de cerrar las brechas analogicas y las digitales", en

B. Solís (coord.), El reto de México ante la Cumbre Mundial de la Sociedad de la Información, Fundación Konrad Adenauer, México, pp. 233-236.

Davara, M. (2000), De las autopistas de la información a la sociedad virtual, Aranzadi Editorial, España.

De Kerchove, D. (2000), Inteligencias en conexión, Gedisa, España.

- (2001), The architecture of intelligence, Birkhauser, Boston.

Drucker, P. (1968), The age of discontinuity: Gutdelines to our cbanging society, New Brunswick, Estados Unidos.

Echeverría, J. (1994), Telépolis, Destino, Barcelona.

Forester, T. (1991), Soctedad de alta tecnología, Siglo XXI, México.

Foucault, M. (1983), Vigilar y castigar, Nacimiento de ta prision, Siglo XXI, México.

Fuentes, C. (1994), Nuevo tiempo mexicano, Aguilar, México.

Gates, B. (1995), The Road Abead, Wamer Books, Estados Unidos.

- (2000): Business the speed of though. Using a digital nervous system, Warner Books, Estados Unidos.

(2002), "La amenaza del sida", en El Unicersal, 19 de noviembre, p. 2.

Gutiérrez, A. (2004), "Chihuahua: la justicia a las urnas", en Proceso, 27 de junio, número 1443, p.29.

Heilemann, J. (2001), Pride before the fall. The trials of Bill Gates and the end of the Microsoft Era, Harper Collins Publishers, Estados Unidos.

Innis H. (1972), Empire and communications, University of Toronto Press, Toronto. (2004), Cbanging concepts of time, Lanham, MD, Rowman \& Littlefield.

INEGI. "Sistema de Trămites Electrónticos Gubernamentales", en Boletín de Política Informática, año XXV, núm. 2, 2002, p. 1.

Joyanes, L. (1997), Cibersociedad. Los retos sociales ante un mundo digital, McGraw Hill, España.

Krauze, E. (1997), La presidencia imperial. Ascenso y caída del sistema politico mexicano (1940-1996), Tusquets, México.

Leer, A. (2001), La visión de los líderes en la era digital, Pearson Educaciónl. México.

Levinson, P. (1995), Learning cyberspace: Essays of the evolution of media and the new education, Anamnesis Press, San Francisco. 
(1999), Digital McLuban. A guide to the information millennitu, Routledge, Londres/Nueva York.

Margáin, J. (2001), "Sistema e-México: convergencia tecnológica con equidad", en Mercado de Valores, mayo.

Mattelart, A. (1977), Multinacionales y sistemas de comunicación: los aparatos ideológicos del imperialismo, Siglo XXI, México.

- (2000), Historia de la utopía planetaria. De la ciudad profética a la sociedad global, Paidos, España.

- (2002), Historia de la sociedad de la información, Barcelona, Siglo XXI.

Mistretta, M. (2002), "Primer Informe de e-Gobierno. Avances más que virtuales", en Information Week, núm. 64, 5 de septiembre, pp. 10-16.

Mitnick, K. y w. Simon (2002), The ant of deception. Controlling the Human Element of Security, Wiley Publishers, Estados Unidos.

McLuhan, M. (1977), La comprensión de los medios como extensiones del bombre, Diana, México.

Meyrowitz, J. (1985), No sense of place. The impact of electronic media on social bebavior, Oxford University Press, Nueva York.

Negroponte, N. (1996), Ser digital, Océano, México.

Olguín, C. (2002), "Entrevista con Bill Gates. México sigue el mismo camino que $\mathrm{EU}^{n}$, en Milenio semanal, núm. 271, 25 de noviembre.

Ong W. (1977), Interfaces of the world. Studies in the etolution of consciousness and culture, Cornell University Press, Nueva York.

Piedras, E. (2003), "Sociedad de Información...¿ Sin información?", en B. Solís (coord.), El reto de Méxtco ante la Cumbre Mundial de la Sociedad de la Información, Fundación Konrad Adenauer, México, pp. 211-223.

Postman, N. (1979), Teacbing as a conserving activity, Delacorte, Nueva York. (1982), The disappearance of cbildbood, Delacorte, Nueva York. (1993), Tecbnopoly. The surrender of culture to tecbnology, Vintage Books, Nueva York.

(1999), Building a bridge to the eighteentb century. How the past can improve our future, Alfred A. Knopf, Nueva York.

Postman N. y C. Weingartner (1969), Teacbing as a subversive activity, Delta, Nueva York.

Riding, Alan (2000), ¿Cambiară México abora?, Joaquín Mortiz, México.

Robinson, S. (2003), "El reto inmediato: reconfigurar el programa e-México", en B. Solís (coord..), El reto de México ante la Cumbre Mundial de la Sociedad de la Información, Fundación Konrad Adenauer, México, pp. 55-63.

Robles, O. (2000), "Evolución de Internet en México y en América Latina", en O. Islas y F. Gutiérrez (coords.) Internet. El medio inteligente, CECSA, México, pp. 3-25.

L (2001), "Recomendaciones a las conclusiones establecidas en los numerales $8,10,11$ y 12 del Foro Avances en la Legislación en Materia de Comercio Electrónico, referente a los nombres de dominio y a las Propuestas Legislativas Relativas a Nombres de Dominio, presentadas por el Grupo GICE", 5 de noviembre.

Rojo P. (2003), Sociedad global y nuevas tecnologias de la información. Los retos de la comunicactón sacial ante la liberación del mercado europeo, Monografias de Ciencias Sociales y de la Comunicación, Universidad Católica San Antonio, España. 
Sánchez, A. (2001), La era de los afectos en internet, Océano, México.

Sotelo Nava. A. (2001), "El e-Gobierno: estrategia para la innovación en el Gobierno Federal", en Mercado de Valores.

Solís, B. (coord.) (2003), El reto de México ante la Cumbre Mundial de la Sociedad de la Información, Fundación Konrad Adenauer, México.

Strate, L., et al. (2002), Communication and cyberspace. Social interaction in an electronic environment, Hampton Press, Nueva York.

Thesing, J. y F. Piess (1999), Globalización, democracia y medios de comunicactón, Konrad Adenauer Stiftung/CIEDLA, Argentina.

Toffler, A. (1981), La tercera ola, Edivisión, México.

Trejo, R. (1994), La nueva alfombra mágica. Usos y mitos de internet. La red sin redes, Los Libros de Fundesco, México. (2003), "El reto de México ante la Cumbre de la Sociedad de la Información", en B. Solís, (coord.), El reto de México ante la Cumbre Mundial de la Sociedad de la Información, Fundación Konrad Adenauer, México, pp. 257 275.

(2004), "Primera fase de la Cumbre Mundial de la S1. Disparidad de enfoques en Ginebra", en Telos, núm. 59, abril-junio, pp. 156-158.

Whitaker, R. (1999), El fin de la privacidad. Cómo la vigilancia total se estä convintiendo en realidad, Paidós, Barcelona. 